\title{
Analytical study of the plasmonic modes of a metal nanoparticle circular array
}

\author{
Kin Hung Fung and C. T. Chan \\ Department of Physics, The Hong Kong University of Science and Technology, Hong Kong, China \\ (Received 27 December 2007; revised manuscript received 7 April 2008; published 15 May 2008)
}

\begin{abstract}
We analyze the plasmonic modes of a metal nanoparticle circular array. Closed-form solutions to the eigenmode problem are presented. For each polarization, the plasmonic mode with the highest quality is found to be in antiphase. The significant suppression of radiative loss can be understood as the cancellation of the dipolar radiation term in the radiative linewidth. The remaining finite radiative linewidth decreases exponentially as the number of particle increases.
\end{abstract}

DOI: 10.1103/PhysRevB.77.205423

PACS number(s): 78.67.Bf, 73.22.Lp, 73.20.Mf, 78.70.-g

\section{INTRODUCTION}

The subwavelength phenomena of electromagnetic waves have attracted considerable research attention in recent years. Plasmonic materials are capable of supporting subwavelength phenomena near optical frequencies, and, as such, they have been the focus of recent research in near-field optics. ${ }^{1,2}$ The tremendous growth of interest in plasmonic materials in the form of metal nanoparticles (MNPs) has been fueled by the significant improvement in fabrication techniques $^{3,4}$ as well as the emergence of many plausible applications for MNPs, such as in biosensors ${ }^{5}$ and surfaceenhanced Raman scattering processes, ${ }^{6,7}$ as subwavelength waveguides $^{8,9}$ and in near-field imaging. ${ }^{10}$

There have been many numerical studies on MNPs with various shapes and arrangements. In particular, it has been shown that MNP circular arrays may serve as electric and magnetic resonators ${ }^{11}$ and they can significantly reduce radiative loss. ${ }^{12}$ In general, due to the complexity of plasmonic modes, analytical studies on them have been few even when dipole approximation can be applied to MNPs in many cases. Markel $^{13}$ obtained the solutions for two and four particles. Citrin $^{12}$ obtained the solution for circular MNP arrays and, by taking into account all dipolar couplings between particles, he showed that the nearest-neighbor tight-binding model cannot be used because it may give noncausal solutions. However, concrete examples and more thorough analytical work on the complicated solutions are necessary for a clear understanding of the plasmonic modes of MNPs. One of the purposes of this paper is to present an accurate analytical solution to this problem by using concrete examples. Since the derivation of a closed-form solution has not been published and there exist typos ${ }^{14}$ in some of the solutions in Ref. 12, we will also present a rigorous derivation of the correct solutions here. By considering the dependence of the actual resonant frequencies on materials and geometry, we will show how the asymptotic forms of the solutions can help us to understand the physical problem. The analytical solution and discussion focus on plasmonic modes that have low radiative loss. Such high-quality plasmonic modes might be useful for good resonators in recently proposed applications. ${ }^{15-17}$

This paper is organized as follows. In Sec. II, the geometries and the material parameters are described. The closedform solutions to the plasmonic eigenmodes are derived in
Sec. III, which are followed by a comparison to existing numerical results. The mode qualities of the high-quality states are also analyzed in Sec. III. Discussions and conclusions are presented in Sec. IV.

\section{GEOMETRIES AND MATERIAL PARAMETERS}

We consider a circular array of spherical MNPs of particle radius $r_{0}$ [see Fig. 1(a)]. Taking the center of the circular array as the origin, the position vectors of the particle centers are

$$
\mathbf{R}_{m}=R_{0} \cos \frac{2 m \pi}{N} \hat{\mathbf{x}}+R_{0} \sin \frac{2 m \pi}{N} \hat{\mathbf{y}}
$$

where $m=0,1,2, \ldots, N-1$ is the particle index, $R_{0}$ is the radius of the circular array, and $N$ is the number of particles. The center-to-center distance between two adjacent particles is $a=2 R_{0} \sin (\pi / N)$. The dielectric function of the MNPs follows Drude's form,

$$
\varepsilon(\omega)=1-\frac{\omega_{p}^{2}}{\omega(\omega+i \gamma)}
$$

where $\omega$ is the angular frequency, $\omega_{p}$ is the plasma frequency, and $\gamma$ is the electron scattering rate. The background medium has a dielectric constant denoted as $\varepsilon_{h}$. We assume that there is no magnetic susceptibility anywhere (i.e., $\mu$ $\left.=\mu_{h}=1\right)$. We restrict ourselves to the situations when the MNPs are not too close together so that $a \geq 3 r_{0}$, which is a customary assumption for the validity of the dipole approximation.

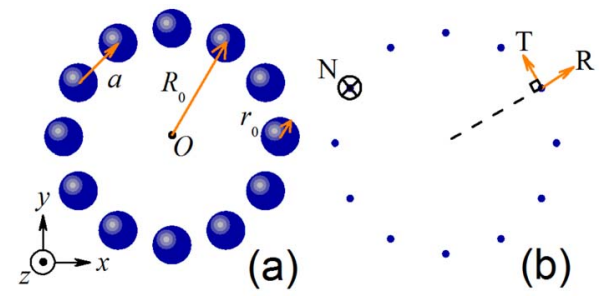

FIG. 1. (Color online) Schematic diagram of the MNP circular array. (a) Geometrical parameters. (b) Polarization vectors. 


\section{EIGENMODE ANALYSIS}

To understand the electromagnetic resonances of an MNP circular array, we solve for the intrinsic normal modes of such a system. The analysis is complicated by the fact that there is always radiation energy loss in a finite array of particles. On top of radiation loss, absorption loss is not negligible in plasmonic systems. To describe the electromagnetic resonances of such systems, a good approach is the eigendecomposition (ED) method $^{13,18,19}$ (sometimes called spectral theory). Bergman and Stroud ${ }^{18}$ proposed such a method to understand the electromagnetic resonances of a finite number of spherical objects. The theory considers all multipolar responses of a spherical object. Later, a simplified version of this method that considers only the dipolar response of small particles was developed by Markel. ${ }^{13}$ The dipolar ED method was successfully applied to systems, such as fractal clusters and periodic MNP arrays. An important advantage of this approach is that it does not require either numerical complex root searching ${ }^{20-22}$ or root approximation. ${ }^{12}$ Here, we will employ the dipolar ED method to analyze the responses of MNP circular arrays. We show that the method allows us to obtain analytical solutions.

\section{A. Dynamic dipole eigenvalue equation}

We consider the case when the particles are well separated $\left(a \geq 3 r_{0}\right)$ such that dipole approximation can already make a good description in optical frequencies. We note that the only approximation that we use in this paper is the dynamic dipole approximation. ${ }^{23,24}$ Retardation effects are included via the dynamic Green's function and material polarizability.

If there is an external time-periodic driving electric field, $\mathbf{E}_{m}^{\mathrm{ext}} e^{-i \omega t}$, acting on the $m$ th particle, the coupled dipole equations can be written as

$$
\mathbf{p}_{m}=\alpha\left[\mathbf{E}_{m}^{\mathrm{ext}}+\sum_{n \neq m} \stackrel{\leftrightarrow}{\mathbf{W}}\left(\mathbf{R}_{m}-\mathbf{R}_{n}\right) \mathbf{p}_{n}\right],
$$

where the dynamic Green's function is ${ }^{13}$

$$
\begin{gathered}
W_{u v}(\mathbf{r})=k_{0}^{3}\left[A\left(k_{0} r\right) \delta_{u v}+B\left(k_{0} r\right) \frac{r_{u} r_{v}}{r^{2}}\right], \\
A(x)=\left(x^{-1}+i x^{-2}-x^{-3}\right) e^{i x}, \\
B(x)=\left(-x^{-1}-3 i x^{-2}+3 x^{-3}\right) e^{i x},
\end{gathered}
$$

$k_{0}=\omega / c, c$ is the speed of light in the background medium, and $u, v=1,2,3$ are component indices in the Cartesian coordinates. Equation (3) can be written as

$$
\sum_{n=1}^{N} \sum_{v=1}^{3} M_{m n u v} p_{n v}=E_{m u}^{\mathrm{ext}},
$$

or $\mathbf{M p}=\mathbf{E}$ in matrix form. Here, $\mathbf{M}$ can be divided into two parts so that

$$
\mathbf{M}=\alpha^{-1} \mathbf{I}-\mathbf{G},
$$

where $\mathbf{I}$ is the identity matrix, and

$$
G_{\text {mnuv }}=\left\{\begin{array}{cc}
W_{u v}\left(\mathbf{r}_{m}-\mathbf{r}_{n}\right), & m \neq n \\
0, & m=n,
\end{array}\right.
$$

which is independent of the material properties. The polarizability $\alpha$ is the dynamic dipole polarizability, ${ }^{23,24}$ which is given by ${ }^{25}$

$$
\alpha(\omega)=i \frac{3 c^{3}}{2 \omega^{3}} a_{1}(\omega)
$$

Here,

$$
a_{1}(\omega)=\frac{q \psi_{1}(q x) \psi_{1}^{\prime}(x)-\psi_{1}(x) \psi_{1}^{\prime}(q x)}{q \psi_{1}(q x) \xi_{1}^{\prime}(x)-\xi_{1}(x) \psi_{1}^{\prime}(q x)}
$$

is the $\ell=1$ electric term of Mie's coefficients, ${ }^{26} \psi_{1}$ and $\xi_{1}$ are the Riccati-Bessel functions, $x=\omega a / c$, and $q=\sqrt{\varepsilon(\omega) / \varepsilon_{h}}$. To analyze the resonances of a cluster of dipoles, we consider the following eigenvalue problem:

$$
\mathbf{M p}=\lambda \mathbf{p}
$$

where $\lambda$ and $\kappa$ are, respectively, the complex eigenvalues of $\mathbf{M}$ and $\mathbf{G}$. These two eigenvalues are related by $\lambda=\alpha^{-1}-\kappa$. The eigenpolarizability is defined as ${ }^{24}$

$$
\alpha_{\text {eig }}=\frac{1}{\lambda} \text {. }
$$

This quantity can be interpreted as the collective response function of the whole system for an external electric field pattern that is proportional to the corresponding eigenvector. Both $\lambda$ and $\alpha_{\text {eig }}$ are useful quantities for analyzing the intrinsic normal modes of the system. Their solutions are given in Secs. III B to III D.

\section{B. Analytical eigensolutions}

In general, the eigenvalue problem [Eq. (12)] cannot be analytically solved when a large number of particles is involved $(N \gg 1) .{ }^{27}$ Even if Eq. (12) can be numerically solved, it is very difficult to choose a particular eigenmode from a bunch of eigenmodes because the number of eigenmodes is proportional to $N$. Nevertheless, the problem can be simplified if a system has a high symmetry. ${ }^{24}$ The circular array that we consider has discrete rotational symmetry, which allows us to obtain analytical results for the eigenvectors and eigenvalues.

In Eq. (12), all dipole moments are written in the same Cartesian coordinates. To solve the eigenvalue problem analytically, it is more convenient to transform the coordinates such that the new vectors after the transformation become

$$
p_{m u}^{\prime}=\sum_{v=1}^{3} \tilde{\Omega}_{m u v} p_{m v}
$$

where 


$$
\tilde{\boldsymbol{\Omega}}_{m}=\left(\begin{array}{ccc}
\cos \frac{2 m \pi}{N} & \sin \frac{2 m \pi}{N} & 0 \\
-\sin \frac{2 m \pi}{N} & \cos \frac{2 m \pi}{N} & 0 \\
0 & 0 & 1
\end{array}\right)
$$

is the local transformation for the $m$ th dipole. With the help of the group theory (the details are given in Appendix A), we divide the problem into $N$ sets of eigenvalue problems,

$$
\tilde{\mathbf{M}}^{\prime(j)} \mathbf{c}^{(j, \sigma)}=\lambda^{(j, \sigma)} \mathbf{c}^{(j, \sigma)},
$$

where $\tilde{\mathbf{M}}^{\prime(j)}$ is a $3 \times 3$ matrix, $j=1,2,3, \ldots, N$, and $\sigma$ $=1,2,3$. The eigenvalues $\lambda$ in Eq. (16) are the same as the values given in Eq. (12). They are given by

$$
\lambda^{(j, \sigma)}=\alpha^{-1}-\kappa^{(j, \sigma)},
$$

where

$$
\begin{gathered}
\kappa^{(j, 1)}=\frac{k_{0}^{3}}{2}\left(\Sigma_{j C}+\sqrt{\Sigma_{j B}^{2}-\Sigma_{j S}^{2}}\right), \\
\kappa^{(j, 2)}=\frac{k_{0}^{3}}{2}\left(\Sigma_{j C}-\sqrt{\Sigma_{j B}^{2}-\Sigma_{j S}^{2}}\right), \\
\kappa^{(j, 3)}=k_{0}^{3} \Sigma_{j A},
\end{gathered}
$$

with the corresponding eigenvectors

$$
\begin{gathered}
\mathbf{c}^{(j, 1)}=\left(\sum_{j B}-\sqrt{\sum_{j B}^{2}-\Sigma_{j S}^{2}},-\Sigma_{j S}, 0\right)^{\mathrm{T}}, \\
\mathbf{c}^{(j, 2)}=\left(\sum_{j B}+\sqrt{\sum_{j B}^{2}-\Sigma_{j S}^{2}},-\Sigma_{j S}, 0\right)^{\mathrm{T}}, \\
\mathbf{c}^{(j, 3)}=(0,0,1)^{\mathrm{T}} .
\end{gathered}
$$

These eigenvectors are related to $\mathbf{p}^{\prime(j, \sigma)}$ through Eq. (A7). Here, we have the following finite sums:

$$
\begin{gathered}
\Sigma_{j A}=\sum_{m=1}^{N-1} A\left(k_{0} D_{m}\right) e^{i 2 \pi j m / N}, \\
\Sigma_{j B}=\sum_{m=1}^{N-1} B\left(k_{0} D_{m}\right) e^{i 2 \pi j m / N}, \\
\Sigma_{j C}=\sum_{m=1}^{N-1}\left[2 A\left(k_{0} D_{m}\right)+B\left(k_{0} D_{m}\right)\right] \cos \frac{2 m \pi}{N} e^{i 2 \pi j m / N}, \\
\sum_{j S}=\sum_{m=1}^{N-1}\left[2 A\left(k_{0} D_{m}\right)+B\left(k_{0} D_{m}\right)\right] \sin \frac{2 m \pi}{N} e^{i 2 \pi j m / N} .
\end{gathered}
$$

Detailed derivations of the results are given in Appendix A. We note that $\Sigma_{j S}$ can be zero when $N$ is even and $j$ $=N / 2, N$. In the case that $\Sigma_{j S}=0$, the first and second eigenvectors should be replaced by $\mathbf{c}^{(j, 1)}=(0,1,0)^{T}$ and $\mathbf{c}^{(j, 2)}$ $=(1,0,0)^{T}$. The corresponding eigenvalues are $\kappa^{(j, 1)}$ $=k_{0}^{3}\left(\Sigma_{j C}+\Sigma_{j B}\right) / 2$ and $\kappa^{(j, 2)}=k_{0}^{3}\left(\Sigma_{j C}-\Sigma_{j B}\right) / 2$.
From Eqs. (21)-(23), we can classify the eigenmodes into parallel-to-plane (PP) modes $(\sigma=1,2)$ and normal-to-plane (NP) modes $(\sigma=3)$. For each $\sigma$, there is one nondegenerate in-phase mode $(j=N)$ for any $N$, and one additional nondegenerate antiphase mode $(j=N / 2)$ for even $N$. Other modes are twofold degenerate. The NP modes are polarized perpendicular to the $x y$ plane, while the PP modes are polarized elliptically in the $x y$ plane, except for $j=N / 2$ and $j=N$, which correspond to linear polarization in the $x y$ plane. The $\sigma=1, j=N$ mode is the in-phase magnetic mode, which is the focus of Ref. 11 and the $\sigma=3, j=N$ mode is the in-phase pure electric radiative mode.

\section{Polarizabilities of the modes}

We derived the closed-form solutions to the eigenvalue problem above. Here, we analyze the eigenmodes using the "eigenpolarizabilities" defined in Eq. (13). For the first concrete example, we take $N=12, r_{0}=25 \mathrm{~nm}, a=75 \mathrm{~nm}, \omega_{p}$ $=9.2 \mathrm{eV}, \gamma=0.1 \mathrm{eV}$, and $\varepsilon_{h}=2.5921$. The material parameters represent approximately the situation of embedding silver nanoparticles in a dielectric. This could be done by placing silver particles on top of, for example, a glass substrate and filling the space with an index-matching fluid. The geometrical parameters are chosen to match those commonly used in the literature so that meaningful comparisons can be made. $^{20,22,24}$ The real and the imaginary parts of $\alpha_{\text {eig }}$ are calculated as a spectral function of frequency using the analytical formulae in Eq. (17). We plot the normalized mode polarizabilities versus the photon energy for the PP and NP modes separately in Figs. 2 and 3, respectively. In each figure, we also include the numerical data calculated by numerical diagonalizations of $\mathbf{M}$ [the matrices are diagonalized using routines in LAPACK (Ref. 28)]. The insets of Figs. 2 and 3 show the enlarged part of the same plot for a higher clarity of the region where there is a high density of data points. The analytical and numerical results are essentially in perfect agreement. There are 14 curves in both Figs. 2(a) and 2(b), while the number of curves in each of Figs. 3(a) and 3(b) are seven. From the analysis in Sec. III B, there should be five twofold-degenerate eigenspaces and two nondegenerate eigenvectors for each $\sigma$. For that reason, the total number of curves (21) is smaller than the matrix dimension, $3 N(=36)$. In spite of the degeneracy, the number of curves linearly grows with the number of particles [i.e., the complexity is $\mathrm{O}\left(N^{3}\right)$ if we diagonalize the matrix with standard numerical packages]. For a large number of particles, it is a difficult task to trace the curves from the mixed data. For example, it would be difficult to connect the points in Figs. 2 and 3 if we did not know the analytical results. The analytical formulas are thus essential for analyzing such systems.

We can see a typical Lorentzian resonant feature for each of the eigenpolarizabilities in Figs. 2 and 3. The real part, $\operatorname{Re}\left(\alpha_{\text {eig }}\right)$, in general exhibits a peak followed by a dip and it changes from positive to negative, while the imaginary part, $\operatorname{Im}\left(\alpha_{\text {eig }}\right)$, is positive definite. The peaks shown in Figs. 2(b) and 3(b) are the resonant (normal mode) frequencies. The corresponding widths of the peaks, which are to be discussed in greater detail in Sec. III D, are inversely proportional to 

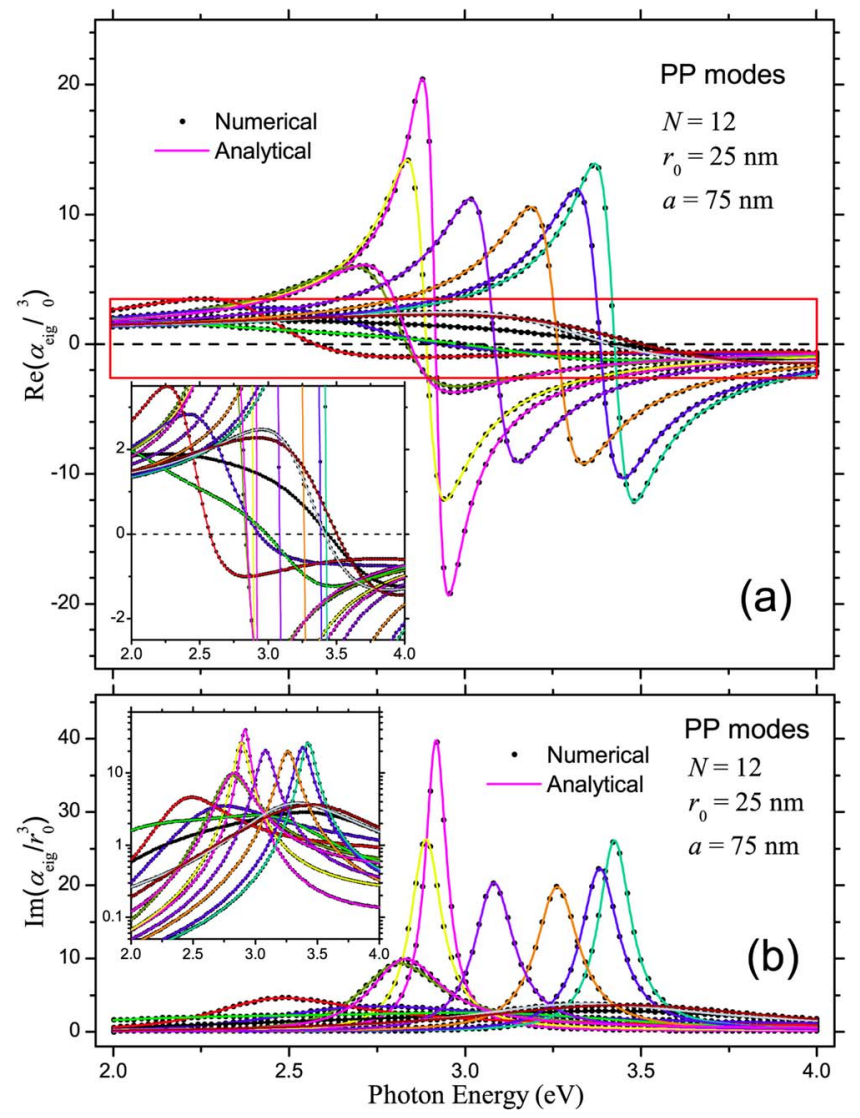

FIG. 2. (Color online) Eigenpolarizability spectra of PP modes. (a) Real part. (b) Imaginary part. In (a), the insets show the enlarged parts of the same figure corresponding to the domain inside the red rectangular box. In (b), the inset shows the same data in the logarithmic scale. The structural and material parameters are $N=12$, $r_{0}=25 \mathrm{~nm}, a=75 \mathrm{~nm}, \omega_{p}=9.2 \mathrm{eV}, \gamma=0.1 \mathrm{eV}$, and $\varepsilon_{h}=2.5921$.

the quality of the system. At first glance, we see a high density of high-quality states from 2.9 to $3.5 \mathrm{eV}$, especially near $2.9 \mathrm{eV}$. These correspond to the higher order modes of the circular array. The two highest peaks near 2.9 and $3.4 \mathrm{eV}$ in Fig. 2 and the highest peak near $2.9 \mathrm{eV}$ in Fig. 3 are the antiphase $(j=N / 2)$ modes of three different polarizations. If the interparticle distances are much smaller than the optical wavelength, these modes can have very narrow linewidths. These are analyzed in Sec. III D. With the analytical solutions, we can now identify all the curves shown in Figs. 2 and 3.

We next consider the NP modes and plot the analytical solutions in Fig. 4(a). It is clear that the antiphase $(j=6)$ mode has the smallest linewidth and the linewidths become larger for other modes of $j$ other than $j=6$. In the following, we consider the effect of the interparticle distance and the particle size. Let us increase the interparticle distance to $a$ $=150 \mathrm{~nm}$, which is twice the first example, but keep the other parameters fixed. The results for the NP modes are shown in Fig. 4(b). In this case, the narrow-linewidth modes disappear. All the eigenmodes have similar spectral features. This is expected since a larger interparticle distance should reduce the interparticle coupling, and the response of each and every eigenmode should go back to that of the single

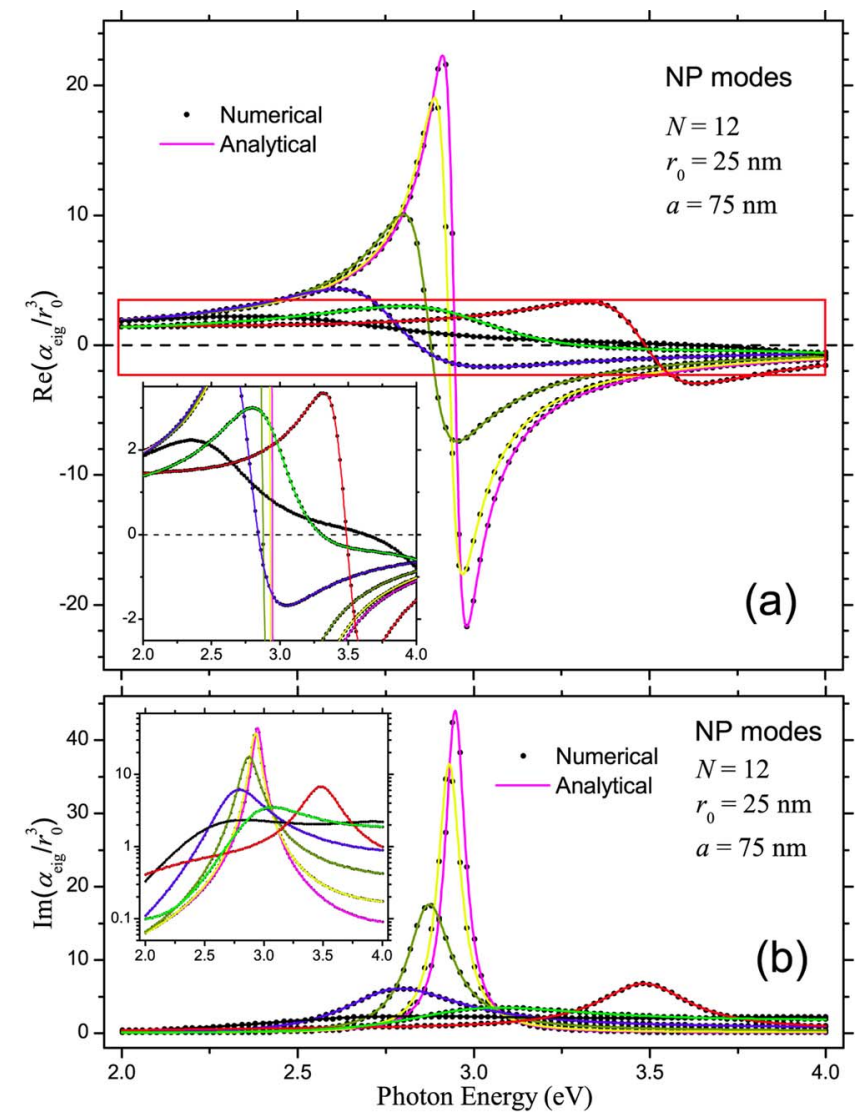

FIG. 3. (Color online) Eigenpolarizability spectra of NP modes. (a) Real part. (b) Imaginary part. In (a), the insets show the enlarged parts of the same figure corresponding to the domain inside the red rectangular box. In (b), the inset shows the same data in the logarithmic scale. The structural and material parameters are the same as Fig. 2.

particle. Once we increase the particle size by twice to $r_{0}$ $=50 \mathrm{~nm}$ so that the interparticle couplings become strong again, the narrow-linewidth modes appear again [see Fig. 4(c)]. The situation in Fig. 4(c) is the same as that of Fig. 4(a), except that there is global geometrical scaling by a factor of 2 . We see that the overall features are the same in Figs. 4(a) and 4(c), except that the resonant frequencies for larger particles are typically lower, and that is tied to the fact that the resonance of a single sphere is shifted to a lower frequency when the size increases. We also observe extra resonances in the higher frequency regime, and these higher harmonics appear because of the large array size.

\section{Mode qualities}

The imaginary part of the eigenpolarizability, $\operatorname{Im}\left(\alpha_{\mathrm{eig}}\right)$, is proportional to the energy extinction. A particular peak frequency of the spectral function, $\operatorname{Im}\left(\alpha_{\mathrm{eig}}\right)$, indicates a resonant frequency $\omega_{0}$ and the corresponding peak width $\delta$ equals the inverse of the relaxation time, $1 / \tau$. The quality factor $Q$ of a particular mode is then given by $\omega_{0} / \delta$. To analytically find $Q$, it is more convenient to find the form of $\operatorname{Im}(\lambda)$, which is related to $\operatorname{Im}\left(\alpha_{\text {eig }}\right)$ by 


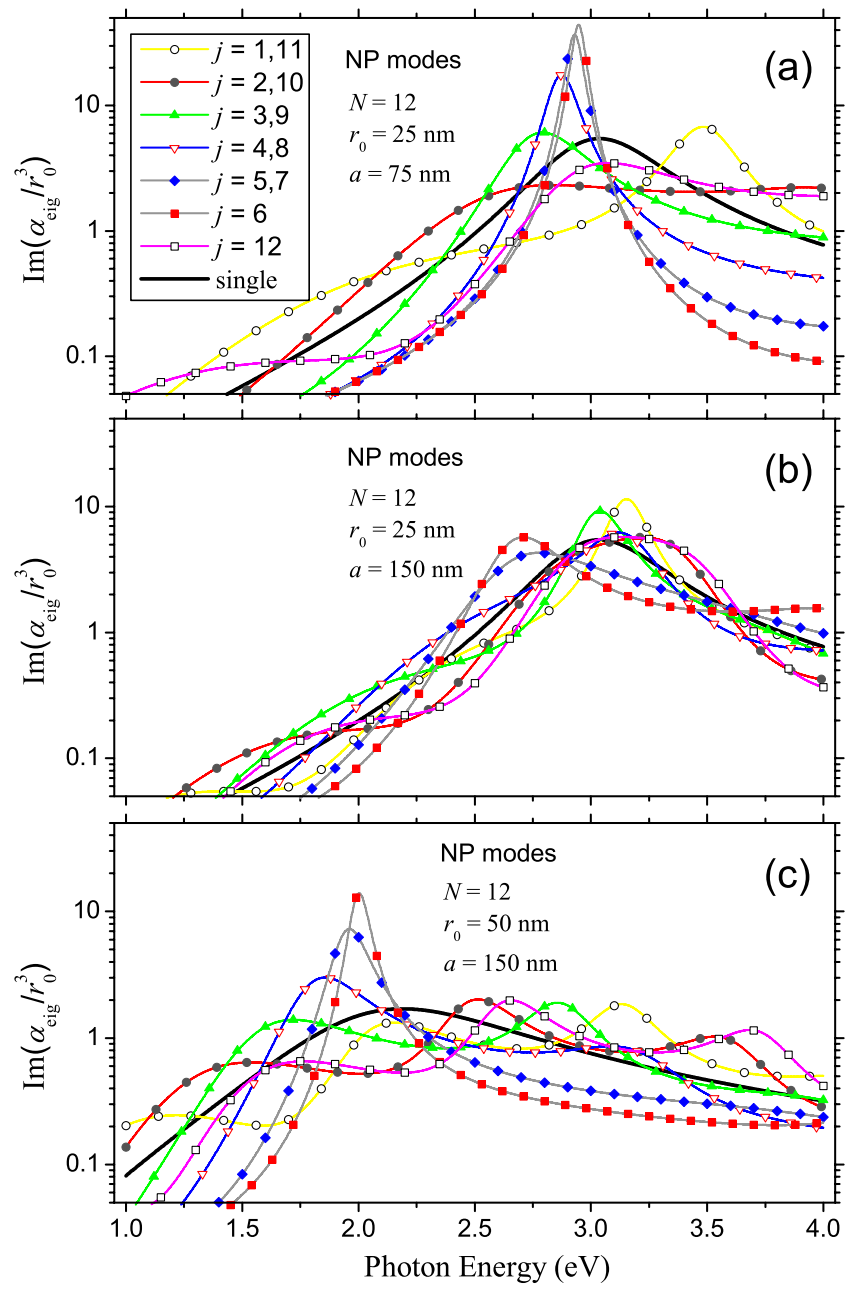

FIG. 4. (Color online) Eigenpolarizability spectra of NP modes. (a) $r_{0}=25 \mathrm{~nm}$ and $a=75 \mathrm{~nm}$. (b) $r_{0}=25 \mathrm{~nm}$ and $a=150 \mathrm{~nm}$. (c) $r_{0}=50 \mathrm{~nm}$ and $a=150 \mathrm{~nm}$. Other parameters are the same as in Fig. 2.

$$
\operatorname{Im}\left(\alpha_{\text {eig }}\right)=-\frac{\operatorname{Im}(\lambda)}{\operatorname{Re}(\lambda)^{2}+\operatorname{Im}(\lambda)^{2}} .
$$

Near the resonant frequency, we can approximate $\operatorname{Re}(\lambda)$ by

$$
\operatorname{Re}[\lambda(\omega)] \approx\left(\omega-\omega_{0}\right) \chi\left(\omega_{0}\right)
$$

where

$$
\chi(\omega)=\frac{d \operatorname{Re}[\lambda(\omega)]}{d \omega}
$$

since $\operatorname{Re}[\lambda(\omega)]$ will cross zero at resonance. ${ }^{29}$ With Eqs. (28) and (29), we thus have

$$
\delta \approx-\frac{\operatorname{Im}\left[\lambda\left(\omega_{0}\right)\right]}{\left|\chi\left(\omega_{0}\right)\right|} .
$$

The function, $\chi(\omega)$, depends very much on the choice of material. In this section, we do not analyze the effect of energy absorption by a particular material and will focus mainly on the suppression of radiation loss. For simplicity, we do not include absorption in the following derivations.
Since $\chi\left(\omega_{0}\right)$ is approximately the same for all modes, $\operatorname{Im}\left[\lambda\left(\omega_{0}\right)\right]$ is usually used to analyze the linewidth. ${ }^{13}$ It can be divided into two parts,

$$
\operatorname{Im}(\lambda)=-\frac{2}{3} k_{0}^{3}-\operatorname{Im}(\kappa)
$$

Here, we have used the fact that $\operatorname{Im}(1 / \alpha)=-2 k_{0}^{3} / 3$ for nonabsorbing particles.

For large $N$, there is no loss of generality to take $N$ to be even. For each polarization $\sigma$, the antiphase mode $(j=N / 2)$, with each pair of nearest dipoles having opposite dipole moments, will have the smallest linewidth in general. We thus focus on the $j=N / 2$ modes. The dipole moments of these modes are linearly polarized along the tangential $(T)$, radial $(R)$, and normal $(N)$ direction for $\sigma=1,2$, and 3, respectively [see Fig. 1(b)]. The corresponding eigenvalues, which are given by Eq. (17), can be written as

$$
\begin{gathered}
\kappa_{T}=k_{0}^{3}\left(\Sigma_{\text {long }}+\Sigma_{\text {cross }}\right), \\
\kappa_{R}=k_{0}^{3}\left(\Sigma_{\text {trans }}+\Sigma_{\text {cross }}\right), \\
\kappa_{N}=k_{0}^{3} \Sigma_{\text {trans }},
\end{gathered}
$$

where $\Sigma_{\text {trans }}, \Sigma_{\text {long }}$, and $\Sigma_{\text {cross }}$ comes from transverse, longitudinal, and crossed interactions. Using the Laurent series expansions of $\Sigma_{\text {trans }}, \Sigma_{\text {long }}$, and $\Sigma_{\text {cross }}$ given in Appendix B, we get

$$
\frac{\operatorname{Im}\left[\kappa_{T}\right]}{k_{0}^{3}}=-\frac{2}{3}+\frac{N^{2}(N+2)}{2^{N-2}(N+1) ! \sin ^{N-2} \frac{\pi}{N}}\left(k_{0} a\right)^{N-2}+\cdots
$$

$$
\begin{gathered}
\frac{\operatorname{Im}\left[\kappa_{R}\right]}{k_{0}^{3}}=-\frac{2}{3}+\frac{N^{2}(N+2)}{2^{N-2}(N+1) ! \sin ^{N-2} \frac{\pi}{N}}\left(k_{0} a\right)^{N-2}+\cdots, \\
\frac{\operatorname{Im}\left[\kappa_{N}\right]}{k_{0}^{3}}=-\frac{2}{3}+\frac{N(N+2)^{2}}{2^{N-1}(N+3) ! \sin ^{N} \frac{\pi}{N}}\left(k_{0} a\right)^{N}+\cdots .
\end{gathered}
$$

[We note that Eqs. (35) and (36) are the same only up to the term associated with $\left(k_{0} a\right)^{N-2}$.] These formulas are generalized versions (for $N$ particles) of the expression derived by Markel for two and four particles. ${ }^{13}$ We see that all of the above expressions have the first term, $-2 / 3$, that can exactly cancel the single dipole radiation term in Eq. (31), i.e., the collective dipolar radiation is suppressed. The remaining higher-order terms contribute to the linewidth. For a fixed $N \gg 1$, as long as $k_{0} a$ is small enough such that the first nonzero higher-order term dominates, the linewidths are $\delta$ $\sim\left(k_{0} a / \pi\right)^{N+1}$ for both tangential and radial modes and $\delta$ $\sim\left(k_{0} a / \pi\right)^{N+3}$ for the normal-to-plane mode. Compared to the linewidth of single dipole resonance, $\delta_{d} \sim\left(k_{0} a\right)^{3}$, the linewidths of these antiphase modes are significantly reduced when $N$ is large. The above approximate forms of the line- 


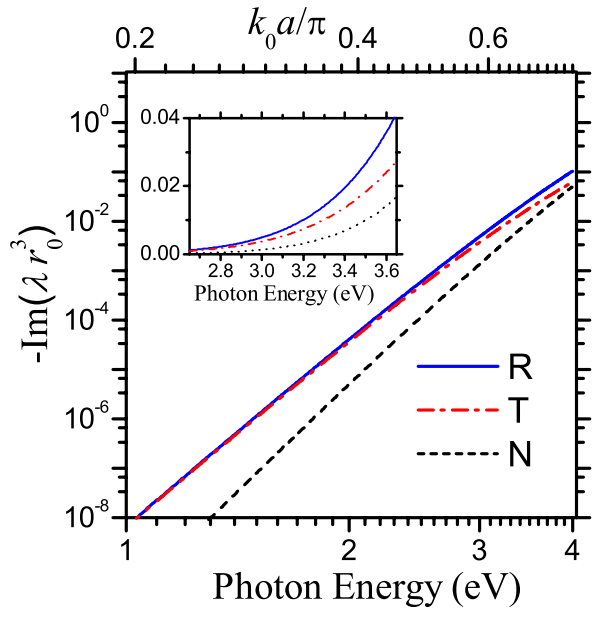

FIG. 5. (Color online) Linewidths of the highest-quality plasmonic modes for each polarization vs resonant frequency. Radial mode $(R)$. Tangential mode $(T)$. Normal-to-plane mode $(N)$. The inset shows an enlarged part in the linear scale. $\gamma=0$.

widths are accurate when $k_{0} a \ll \pi$. For an example, we take the same parameters as in Sec. III C except for $\gamma=0$. Figure 5 shows a plot of the exact analytical value of $-\operatorname{lm}\left(\lambda r_{0}^{3}\right)$ [given by Eq. (17)] against resonant frequency. At low frequency $\left(k_{0} a<0.4 \pi\right)$, the graph (in log-log scale) shows straight lines with slopes that are consistent with the approximated power factor, $N+1$, for tangential and radial modes and $N+3$ for the normal-to-plane mode. The overlapping between the tangential and radial modes also agrees with the expressions in Eqs. (35) and (36). The lines bend slightly downward at higher frequencies $\left(k_{0} a>0.4 \pi\right)$, indicating that the linewidths are smaller than the approximated power laws. In this range, the tangential and radial modes also start to separate from each other. In general, the linewidth of a resonant state rapidly decreases as the resonant frequency decreases. The actual resonant frequencies fall in the range of $0.4 \pi<k_{0} a<0.7 \pi$ for our material parameters. By inspecting the interaction of surface charges ${ }^{30}$ on the MNPs, we know that the normal-to-plane and radial modes have similar resonant frequencies that are lower than the single sphere resonant frequency, while the tangential mode is higher. Therefore, this suggests that the linewidth of the tangential mode is much larger than that of the radial mode although their linewidths have the same form $\left[\delta \sim\left(k_{0} a / \pi\right)^{N+1}\right]$. Furthermore, the linewidth of the normal-to-plane mode is smaller than that of the tangential mode by a factor of $\left(k_{0} a / \pi\right)^{2}$ approximately. Figure 6 shows the exact analytical values of $\operatorname{Im}\left(\alpha_{\text {eig }}\right)$ for these three modes. Figure 6 shows sharp peaks that agree with our estimation in terms of both peak frequency and peak width. Here, we conclude that the best quality mode is the antiphase NP mode with a linewidth that takes the form of $\delta \sim\left(k_{0} a / \pi\right)^{N+3}$.

Although $N$ is assumed to be even in the above analysis, the general properties of the linewidths are almost the same for odd $N$. However, the linewidth of the highest quality state for odd $N$ is $\delta \sim\left(k_{0} a / \pi\right)^{N+2}$ instead. Another difference is that the highest quality state(s) for even $N$, i.e., the $j=N / 2$ state, is nondegenerate, while that for odd $N$, i.e., the $j$ $=(N \pm 1) / 2$ states, are twofold degenerate. Figure 7 shows

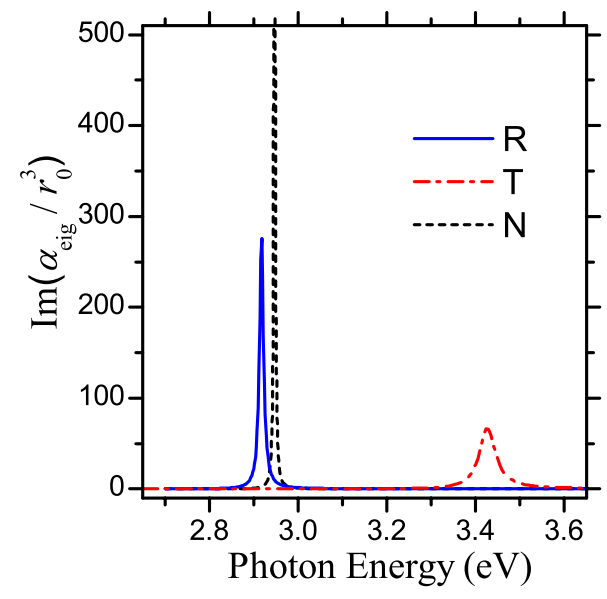

FIG. 6. (Color online) $\operatorname{Im}\left(\alpha_{\text {eig }}\right)$ of the highest-quality plasmonic modes for each polarization vs photon energy. Radial mode $(R)$. Tangential mode $(T)$. Normal-to-plane mode $(N) . \gamma=0$.

the linewidths [given by Eq. (17)] of the highest quality modes against resonant frequency for different numbers of particles. We see that the linewidths increase with the resonant frequency in the form very close to our predicted power laws. For a given even number $N_{0}$, the linewidths of the $N$ $=N_{0}$ case and the $N=N_{0}-1$ case nearly overlap. In addition, it is shown in Fig. 8 that the linewidths decay exponentially as $N$ increases (separately for even and odd numbers).

\section{DISCUSSION AND CONCLUSION}

In summary, we have rigorously derived the analytical eigensolutions to the plasmonic modes of MNP circular arrays within the dynamic dipole approximation. Significant suppression of radiation loss was found in some of the eigenmodes. The high-quality plasmonic modes were understood via the analytical solutions. We found that the antiphase mode could almost cancel the radiative linewidth of a single dipole. The normal-to-plane antiphase mode was found to be the highest-quality mode among all modes. If the resonant

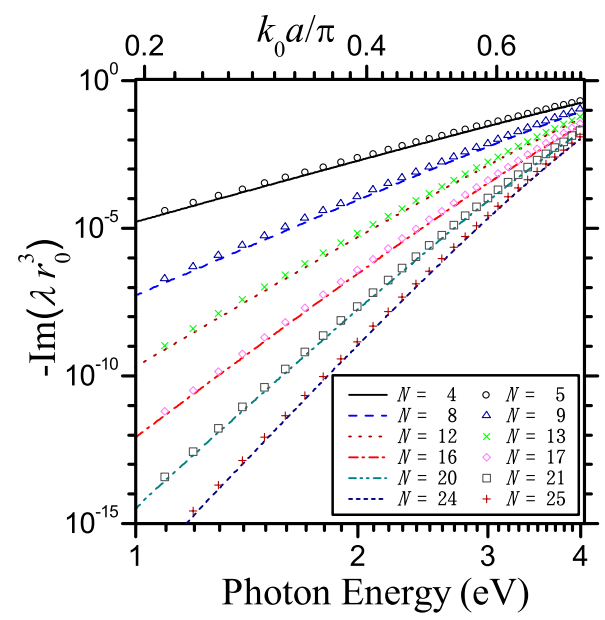

FIG. 7. (Color online) Linewidths of the anti-phase normal-toplane mode vs resonant frequency. $\gamma=0$. 


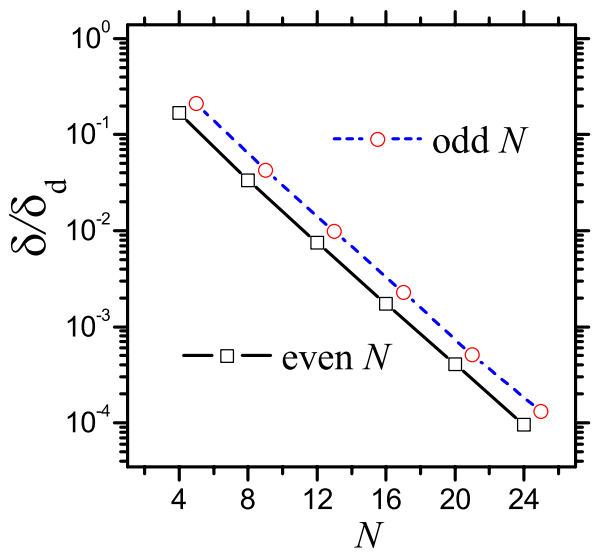

FIG. 8. (Color online) Linewidths of the anti-phase normal-toplane mode vs number of particles in the ring. $\delta_{d}$ is the linewidth of a single particle. $\gamma=0$.

frequency is low $\left(k_{0} a<\pi\right)$, the remaining radiative linewidth was found to be dominated by a term proportional to $\left(k_{0} a / \pi\right)^{N+3}$ for even numbers of particles $N$. Our solutions could be useful in understanding the complicated response properties of MNP circular arrays and wave propagation in MNP plasmonic waveguides with bending. Our results here are based on local theory, but one could easily rederive the results using nonlocal theory, provided that a valid nonlocal dielectric function is given.

\section{ACKNOWLEDGMENTS}

This work was supported by the Central Allocation Grant from the Hong Kong RGC through HKUST 3/06C. Computation resources were supported by the Shun Hing Education and Charity Fund. We thank Jianwen Dong and Dezhuan Han for useful discussions.

\section{APPENDIX A: DERIVATION OF THE ANALYTICAL SOLUTION}

The transformation given by Eq. (14) can be written, globally, as

$$
\mathbf{p}^{\prime}=\mathbf{\Omega} \mathbf{p},
$$

where

$$
\boldsymbol{\Omega}=\left(\begin{array}{ccccc}
\tilde{\boldsymbol{\Omega}}_{1} & \mathbf{O} & \mathbf{O} & \mathbf{O} & \mathbf{O} \\
\mathbf{O} & \tilde{\boldsymbol{\Omega}}_{2} & \mathbf{O} & \mathbf{O} & \mathbf{O} \\
\mathbf{O} & \mathbf{O} & \tilde{\boldsymbol{\Omega}}_{3} & \mathbf{O} & \mathbf{O} \\
\mathbf{O} & \mathbf{O} & \mathbf{O} & \ddots & \vdots \\
\mathbf{O} & \mathbf{O} & \mathbf{O} & \cdots & \tilde{\boldsymbol{\Omega}}_{N}
\end{array}\right) .
$$

The transformed eigenvalue problem becomes

$$
\mathbf{M}^{\prime} \mathbf{p}^{\prime}=\lambda \mathbf{p}^{\prime},
$$

where $\mathbf{M}^{\prime}=\mathbf{\Omega} \mathbf{M} \boldsymbol{\Omega}^{-1}$. Due to the discrete rotational symmetry of the system, $\mathbf{M}^{\prime}$ is invariant under a cyclic index trans- lation, $m \rightarrow \operatorname{Mod}(m+n, N)$, i.e., $\mathbf{T}(n) \mathbf{M}^{\prime} \mathbf{T}(n)^{-1}=\mathbf{M}^{\prime}$, for $n$ $=1,2,3, \ldots, N$, where

$$
\mathbf{T}(n)=\left(\begin{array}{ccccc}
\mathbf{O} & \tilde{\mathbf{I}} & \mathbf{O} & \mathbf{O} & \mathbf{0} \\
\mathbf{O} & \mathbf{O} & \tilde{\mathbf{I}} & \ddots & \vdots \\
\mathbf{O} & \mathbf{O} & \mathbf{O} & \ddots & \mathbf{0} \\
\mathbf{O} & \mathbf{O} & \mathbf{O} & \ddots & \tilde{\mathbf{I}} \\
\tilde{\mathbf{I}} & \mathbf{O} & \mathbf{O} & \cdots & \mathbf{O}
\end{array}\right),
$$

and $\tilde{\mathbf{I}}$ is a $3 \times 3$ identity matrix. In other words, $\mathbf{T}(n)$ and $\mathbf{M}^{\prime}$ commute. We note that the set, $\{\mathbf{T}(1), \mathbf{T}(2), \mathbf{T}(3), \ldots, \mathbf{T}(N)\}$, form a cyclic group $\mathrm{C}_{N}$. The simultaneous eigenvectors of the group and the corresponding eigenvalues of $\mathbf{T}(n)$ are denoted by $\mathbf{v}^{(j)}$ and $t_{n}^{(j)}$, respectively, with

$$
t_{n}^{(j)}=e^{i 2 \pi j n / N}
$$

and

$$
v_{m u}^{(j)}=c_{u}^{(j)} e^{i 2 \pi j m / N},
$$

where $c_{1}^{(j)}, c_{2}^{(j)}$, and $c_{3}^{(j)}$ are arbitrary constants and $j$ $=1,2,3 \ldots, N$. The eigenvectors $\mathbf{v}^{(j)}$ of $\mathbf{T}(n)$ are also the eigenvectors of $\mathbf{M}^{\prime}$. Therefore, we can write the eigenvectors $\mathbf{p}^{\prime(j, \sigma)}$ of $\mathbf{M}^{\prime}$ in the form of

$$
p_{m u}^{\prime(j, \sigma)}=c_{u}^{(j, \sigma)} e^{i 2 \pi j m / N},
$$

where $\sigma$ is an index allowing further reduced eigenspaces. After substituting Eq. (A7) into Eq. (A3), the eigenvalue problem becomes

$$
\sum_{v=1}^{3} \tilde{M}_{u v}^{(j)} c_{v}^{(j, \sigma)}=\lambda^{(j, \sigma)} c_{u}^{(j, \sigma)}
$$

where

$$
\tilde{M}_{u v}^{\prime(j)}=\sum_{m=1}^{N} M_{N m u v}^{\prime} e^{i 2 \pi j m / N} .
$$

If we write Eq. (A8) explicitly in a matrix form, we will finally get the $3 \times 3$ matrix equation,

$$
\tilde{\mathbf{M}}^{\prime(j)} \mathbf{c}^{(j, \sigma)}=\lambda^{(j, \sigma)} \mathbf{c}^{(j, \sigma)},
$$

where

$$
\begin{gathered}
\tilde{\mathbf{M}}^{(j)}=\alpha^{-1} \mathbf{I}-\tilde{\mathbf{G}}^{(j)}, \\
\widetilde{\mathbf{G}}^{(j)}=k_{0}^{3} \sum_{m=1}^{N-1}\left[A\left(k_{0} D_{m}\right) \tilde{\mathbf{\Omega}}_{m}^{-1}+B\left(k_{0} D_{m}\right)\left(\widetilde{\mathbf{\Omega}}_{m}^{-1}+\mathbf{K}\right) / 2\right] e^{i 2 \pi j m / N}, \\
D_{m}=2 R \sin (m \pi / N) \text {, and } \\
\mathbf{K}=\left(\begin{array}{ccc}
-1 & 0 & 0 \\
0 & 1 & 0 \\
0 & 0 & -1
\end{array}\right) .
\end{gathered}
$$

The eigenvalue problem can now be solved in closed form and the results are given by Eqs. (17)-(23). 


\section{APPENDIX B: SERIES EXPANSIONS OF EIGENVALUES}

For the antiphase modes $(j=N / 2)$, the eigenvalues of $\mathbf{G}$ can be divided into the transverse $\left(\Sigma_{\text {trans }}\right)$, longitudinal $\left(\Sigma_{\text {long }}\right)$, and cross-interaction $\left(\Sigma_{\text {cross }}\right)$ terms, as shown in Eqs. (32)-(34). Using Laurent series expansion, we get

$$
\begin{aligned}
& \Sigma_{\text {trans }}=\sum_{n=-3}^{\infty} \frac{(n+2)^{2}}{(n+3) !} \frac{i^{n+1} S_{N, n}}{\sin ^{n} \frac{\pi}{N}}\left(k_{0} a\right)^{n}, \\
& \Sigma_{\text {long }}=\sum_{n=-3}^{\infty} \frac{2(n+2)}{(n+3) !} \frac{i^{n+1} S_{N, n}}{\sin ^{n} \frac{\pi}{N}}\left(k_{0} a\right)^{n},
\end{aligned}
$$

$$
\Sigma_{\text {cross }}=\sum_{n=-3}^{\infty} \frac{(n+2)(n+4)}{2(n+3) !} \frac{i^{n+3} S_{N, n+2}}{\sin ^{n} \frac{\pi}{N}}\left(k_{0} a\right)^{n},
$$

where

$$
S_{N, n}=\sum_{m=1}^{N-1}(-1)^{m} \sin ^{n} \frac{m \pi}{N}
$$

If we take the imaginary part of the eigenvalues, we only leave the terms with $n=0,2,4, \ldots$. Furthermore, for these even terms, we have

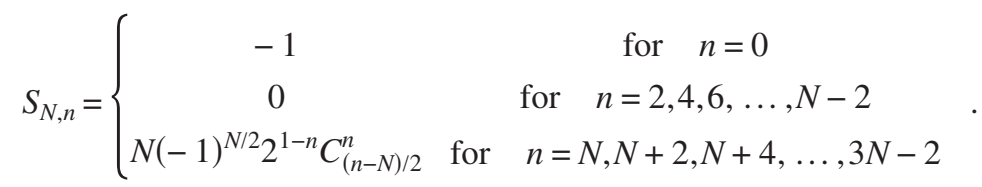

The imaginary parts of the eigenvalues can now be simplified as in Eqs. (35)-(37).

${ }^{1}$ W. L. Barnes, A. Dereux, and T. W. Ebbesen, Nature (London) 424, 824 (2003).

${ }^{2}$ C. Girard, Rep. Prog. Phys. 68, 1883 (2005).

${ }^{3}$ U. Kreibig and M. Vollmer, Optical Properties of Metal Clusters (Springer, Berlin, 1995).

${ }^{4}$ Metal Nanoparticles: Synthesis, Characterication, and Applications, edited by D. L. Feldheim and C. A. Foss, Jr. (Dekker, New York, 2002).

${ }^{5}$ A. J. Haes and R. P. V. Duyne, Anal. Bioanal. Chem. 379, 920 (2004).

${ }^{6}$ M. Moskovits, Rev. Mod. Phys. 57, 783 (1985).

${ }^{7}$ G. Bachelier and A. Mlayah, Phys. Rev. B 69, 205408 (2004).

${ }^{8}$ S. A. Maier, P. G. Kik, H. A. Atwater, S. Meltzer, E. Harel, B. E. Koel, and A. A. G. Requicha, Nat. Mater. 2, 229 (2003).

${ }^{9}$ M. Quinten, A. Leitner, J. R. Krenn, and F. R. Aussenegg, Opt. Lett. 23, 1331 (1998).

${ }^{10}$ C. R. Simovski, A. J. Viitanen, and S. A. Tretyakov, J. Appl. Phys. 101, 123102 (2007).

${ }^{11}$ A. Alù, A. Salandrino, and N. Engheta, Opt. Express 14, 1557 (2006).

${ }^{12}$ D. S. Citrin, J. Opt. Soc. Am. B 22, 1763 (2005).

${ }^{13}$ V. A. Markel, J. Opt. Soc. Am. B 12, 1783 (1995).

${ }^{14}$ There are incorrect signs in Eq. (7) of Ref. 12.

${ }^{15}$ D. J. Bergman and M. I. Stockman, Phys. Rev. Lett. 90, 027402
(2003).

${ }^{16}$ K. Li, X. T. Li, M. I. Stockman, and D. J. Bergman, Phys. Rev. B 71, 115409 (2005).

${ }^{17}$ P. Mühlschlegel, H.-J. Eisler, O. J. F. Martin, B. Hecht, and D. W. Pohl, Science 308, 1607 (2005).

${ }^{18}$ D. J. Bergman and D. Stroud, Phys. Rev. B 22, 3527 (1980).

${ }^{19}$ L. Zhou and S. T. Chui, Phys. Rev. B 74, 035419 (2006).

${ }^{20}$ W. H. Weber and G. W. Ford, Phys. Rev. B 70, 125429 (2004).

${ }^{21}$ A. L. Burin, Phys. Rev. E 73, 066614 (2006).

${ }^{22}$ A. F. Koenderink and A. Polman, Phys. Rev. B 74, 033402 (2006).

${ }^{23}$ V. A. Markel, J. Phys. B 38, L115 (2005).

${ }^{24}$ K. H. Fung and C. T. Chan, Opt. Lett. 32, 973 (2007).

${ }^{25}$ C. E. Dungey and C. F. Bohren, J. Opt. Soc. Am. A 8, 81 (1991).

${ }^{26}$ C. F. Bohren and D. R. Huffman, Absorption and Scattering of Light by Small Particles (Wiley, New York, 1983).

${ }^{27}$ V. A. Markel, J. Phys.: Condens. Matter 18, 11149 (2006).

${ }^{28}$ E. Anderson, Z. Bai, C. Bischof, L. S. Blackford, J. Demmel, J. J. Dongarra, J. D. Croz, S. Hammarling, A. Greenbaum, A. McKenney, and D. Sorensen, LAPACK Users' Guide, 3rd ed. (Society for Industrial and Applied Mathematics, Philadelphia, 1999).

${ }^{29}$ V. A. Markel and E. Y. Poliakov, Philos. Mag. B 76, 895 (1997).

${ }^{30}$ K. H. Fung and C. T. Chan, Opt. Commun. 281, 855 (2008). 